\title{
The Association between Marital Transitions, Body Mass Index, and Weight: A Review of the Literature
}

\author{
Lauren Dinour, ${ }^{1}$ May May Leung, ${ }^{2}$ Gina Tripicchio,, ${ }^{2}$ Sahar Khan,, ${ }^{2}$ and Ming-Chin Yeh$^{2}$ \\ ${ }^{1}$ Department of Public Health, The Graduate Center, City University of New York, 365 Fifth Avenue, New York, NY 10016, USA \\ ${ }^{2}$ City University of New York School of Public Health at Hunter College, 2180 Third Avenue, New York, NY 10035, USA
}

Correspondence should be addressed to Lauren Dinour, ldinour@gc.cuny.edu

Received 1 October 2011; Revised 24 March 2012; Accepted 13 May 2012

Academic Editor: Jack A. Yanovski

Copyright ( $) 2012$ Lauren Dinour et al. This is an open access article distributed under the Creative Commons Attribution License, which permits unrestricted use, distribution, and reproduction in any medium, provided the original work is properly cited.

\begin{abstract}
Objective. To examine the association between different marital transitions and changes in body mass index (BMI) and body weight. Methods. A systematic literature search was conducted for peer-reviewed articles published between January 1990 and December 2011. Longitudinal studies were included if they compared dependent variables, such as BMI or weight, before and after a change in marital status. Results. Twenty articles were included: 4 articles described only transitions into marriage and/or cohabitation, 2 articles described only transitions out of marriage and/or cohabitation, and 14 articles described both. Overall, transitions into marriage were associated with weight gain, whereas transitions out of marriage were associated with weight loss. No major differences were observed between genders or across specific marital transition states. Conclusions. Additional research is warranted to better understand this phenomenon and the impact of marital transitions on obesity and obesity-related behaviors. This paper highlights potential opportunities to incorporate programs, practices, and policies that aim to promote and support healthy weights and lifestyles upon entering or leaving a marriage or cohabiting relationship.
\end{abstract}

\section{Introduction}

Obesity is a major public health issue, both nationally and internationally. Recent reports estimate that $35.7 \%-$ approximately 72.5 million - of American adults are obese (defined as body mass index $\left.(B M I) \geq 30 \mathrm{~kg} / \mathrm{m}^{2}\right)[1,2]$. Globally in the year 2000, the number of adults with excessive weight exceeded the number who were underweight for the first time in history [3]. The World Health Organization reported that in 2008, 1.5 billion adults 20 years and older were overweight (defined as BMI $\geq 25 \mathrm{~kg} / \mathrm{m}^{2}$ ), and of those, 200 million men and nearly 300 million women were obese [4]. Additionally, obesity is associated with chronic diseases that increase rates of morbidity and mortality, including type 2 diabetes, cardiovascular disease, stroke, hypertension, and certain types of cancer [5].

Obesity is a consequence of multiple factors, and it is important to better understand these factors in order to identify opportunities to promote healthy behaviors, thus reducing the risk of developing obesity and related chronic diseases. One area of research has shown that marriage may be associated with body weight and obesity-related behaviors among adults [6]. For example, research investigating the influences on individual food decisions has found that, among married couples at different stages of the life course, spousal influence was consistently rated as one of the most important sources of influence on individual food decisions $[7,8]$.

While some studies have found that current marital status is itself correlated with body weight and obesity [9], other research suggests that marital transition-the act of moving from one marital status to another-is also important in predicting body weight changes and the behavioral risk factors associated with weight gain [10]. Although researchers note that there are important differences between marriage and cohabitation-living with, but not legally married to, a romantic partner - that may not be associated with the same health benefits seen among the married [11, 12 ], cohabitation is becoming a more commonly occurring relationship status [13] and deserves further investigation. Marital transition is a relevant target for obesity research as most adults marry or cohabit [14]. Therefore, there 
is a need to evaluate the body of literature focusing on marital transitions, both into and out of marriage and/or cohabitation, and their associations with body weight change [15].

Marital transition has been defined as a change in marital status during a given time period that occurs due to entry into marriage, exit out of marriage as a result of divorce or spousal death, and remarriage following divorce or spousal death [16]. In addition, since cohabiting can be considered a relationship status different from married, we broaden the definition of marital transition to include transitions into and out of cohabitation. The current review evaluates changes in BMI and body weight as a result of marital transition. Specific transitions into and out of marriage, including cohabitation, remarriage, divorce or separation, and widowhood, are independently evaluated to provide further clarity on the differences in body weight trends as a result of these transitions. The findings from this review highlight areas for further research, as well as opportunities to incorporate new programs, practices, and policies that seek to promote healthy weights and behaviors upon entering or leaving a marriage or cohabiting relationship.

\section{Methods}

2.1. Literature Search. Ten databases (Academic Search Complete, Anthropology Plus, CINAHL and CINAHL Plus, EconLit, Medline, PsychInfo, Scopus, SocIndex, Sociological Abstracts, and Web of Science) were searched for the relevant articles published between January 1990 and December 2011. These databases were searched because they provide most comprehensive scholarly and multidisciplinary abstracts relevant to this area of research. For this review keywords such as "marri*," "marry*," "marital," "divorc*," "widow*," "cohabit*," "weight change," "weight gain," "weight loss," "overweight," "obes*," "body mass index," "BMI," "cohort," "longitudinal," "adult," "m?n," and "wom?n" were used in combination, where ${ }^{*}$ and ? signify truncation and wildcard searches, respectively (to account for various endings or multiple spellings). Relevant references were extracted and examined, and a list was compiled in the form of titles and abstracts of the selected articles.

2.2. Inclusion Criteria. Articles were included if they were longitudinal in design and compared a weight-related dependent variable before and after a change in marital status. Marital status was defined as an individual's current relationship state, such as never married, cohabiting, married, divorced/separated, or widowed; whereas marital transition was defined as a change in marital status over time. Transitions into marriage could have included never married to married, never married to cohabiting, cohabiting to married, divorced/separated or widowed to remarried, and divorced/separated or widowed to cohabiting. Transitions out of marriage could have included cohabiting to not living with a romantic partner, married to divorced/separated, and married to widowed. It should be noted that additional marital categories have been identified and reported in the literature, including single, unmarried, and not married. These terms are not precise, as they do not distinguish between never married, divorced/separated, and widowed individuals. However, the purpose of our review was to synthesize the existing literature. In order to accurately reflect the sample of articles included in this review, all terminology reported in the current paper was explicitly used by the authors of the original articles.

Articles were limited to those appearing in peer-reviewed journals. Additional articles that met the above criteria were obtained from reference lists of those retrieved from the database searches and from enquiry with researchers in the field.

2.3. Exclusion Criteria. Searches were conducted only in the English language; all other languages were excluded.

2.4. Selection Process. The results of the preliminary search were reviewed and relevant titles with abstracts were then retrieved. Abstracts that appeared more than once in different databases were removed. Full articles of relevant abstracts were retrieved for further review.

Two authors independently assessed the retrieved articles for inclusion based upon the criteria listed above. Any inconsistencies were resolved by discussions with the other author. A summary table (Table 1) was composed of the selected articles. The table includes characteristics of the participants (sample size, age range, race, response rate, country), dataset from which the data came (if applicable), data collection methods, time parameters of the study or survey (year and length of the study or survey), marital transition(s) studied, key findings, and limitations as reported in the articles.

\section{Results}

A total of 1,190 abstracts were retrieved through the initial search process; 524 were excluded due to repeats, resulting in 666 abstracts identified through the initial search process. Upon review of these abstracts by two separate authors, 38 articles were identified as potentially meeting the inclusion and exclusion criteria. After reviewing the full articles, the two authors agreed that 12 articles met the criteria for inclusion in this review. Five additional articles were identified from the reference lists of the original 12 articles, and 3 more were found through the process of enquiring with researchers in the field. Thus, a final total of 20 articles were included in this review. Figure 1 outlines the results at each stage of the search process.

It should be noted that 8 out of the final 20 articles analyzed data from the same national survey or study as one or more other articles, albeit using different analytic samples (e.g., the final sample size included in the analysis), comparison groups, outcome variables, and/or baseline or follow-up time points $[6,10,22,24-26,32,33]$. For example, Kahn and Williamson [24, 26], Kahn et al. [25], and Sobal et al. [6] analyzed data from the National Health and Nutrition Examination Survey (NHANES I) and the NHANES I Epidemiological Follow-up Study (NHEFS), 


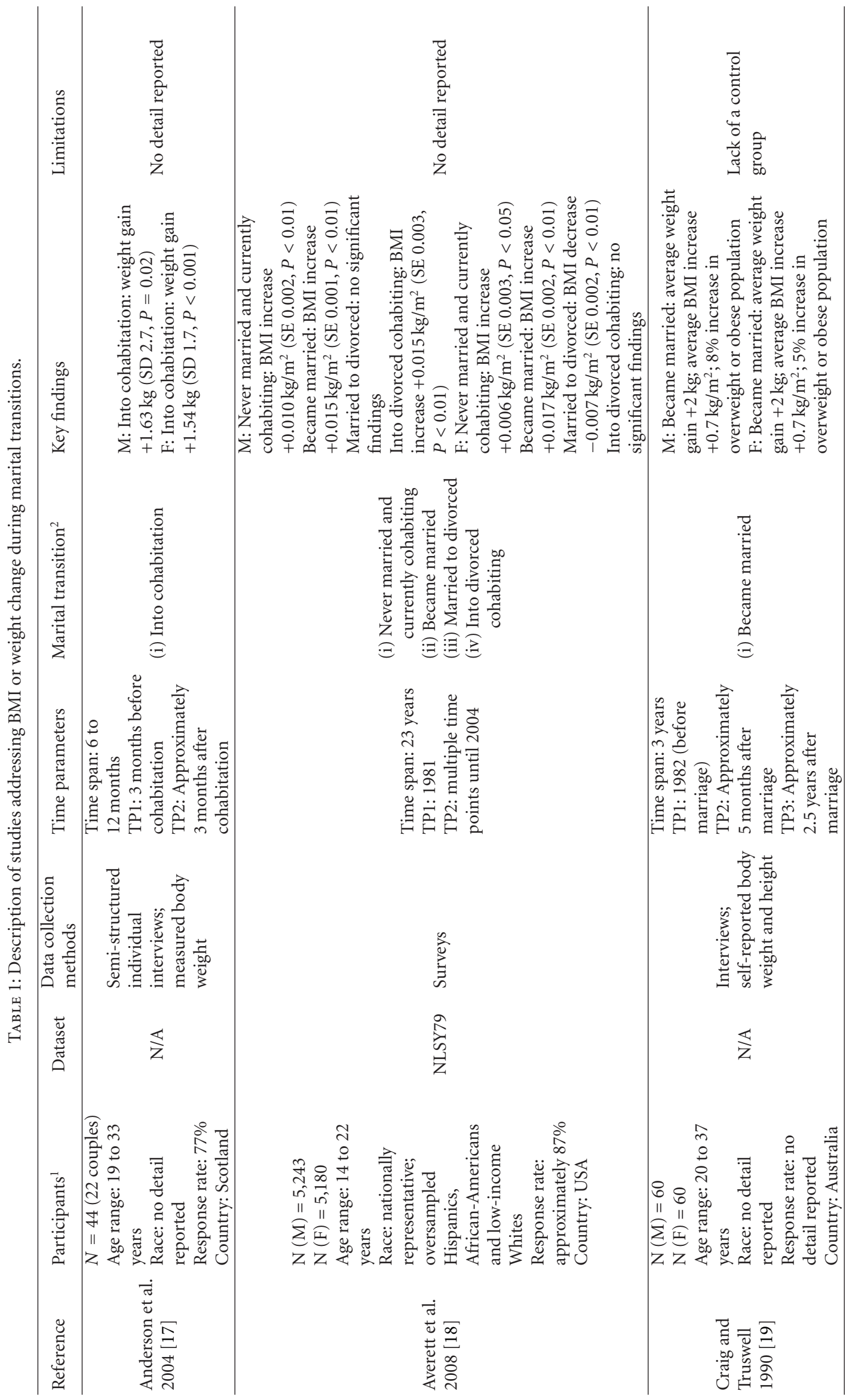




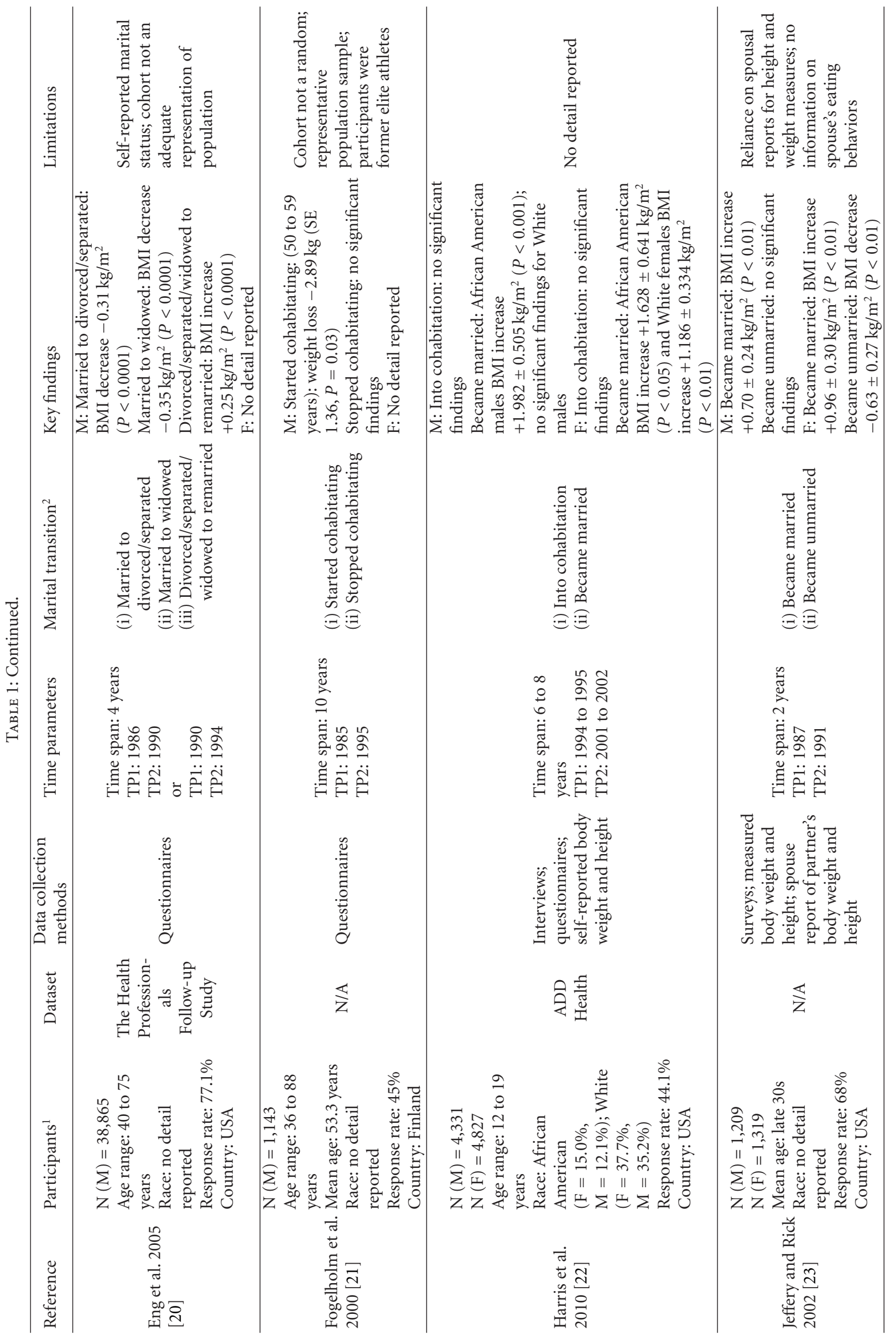




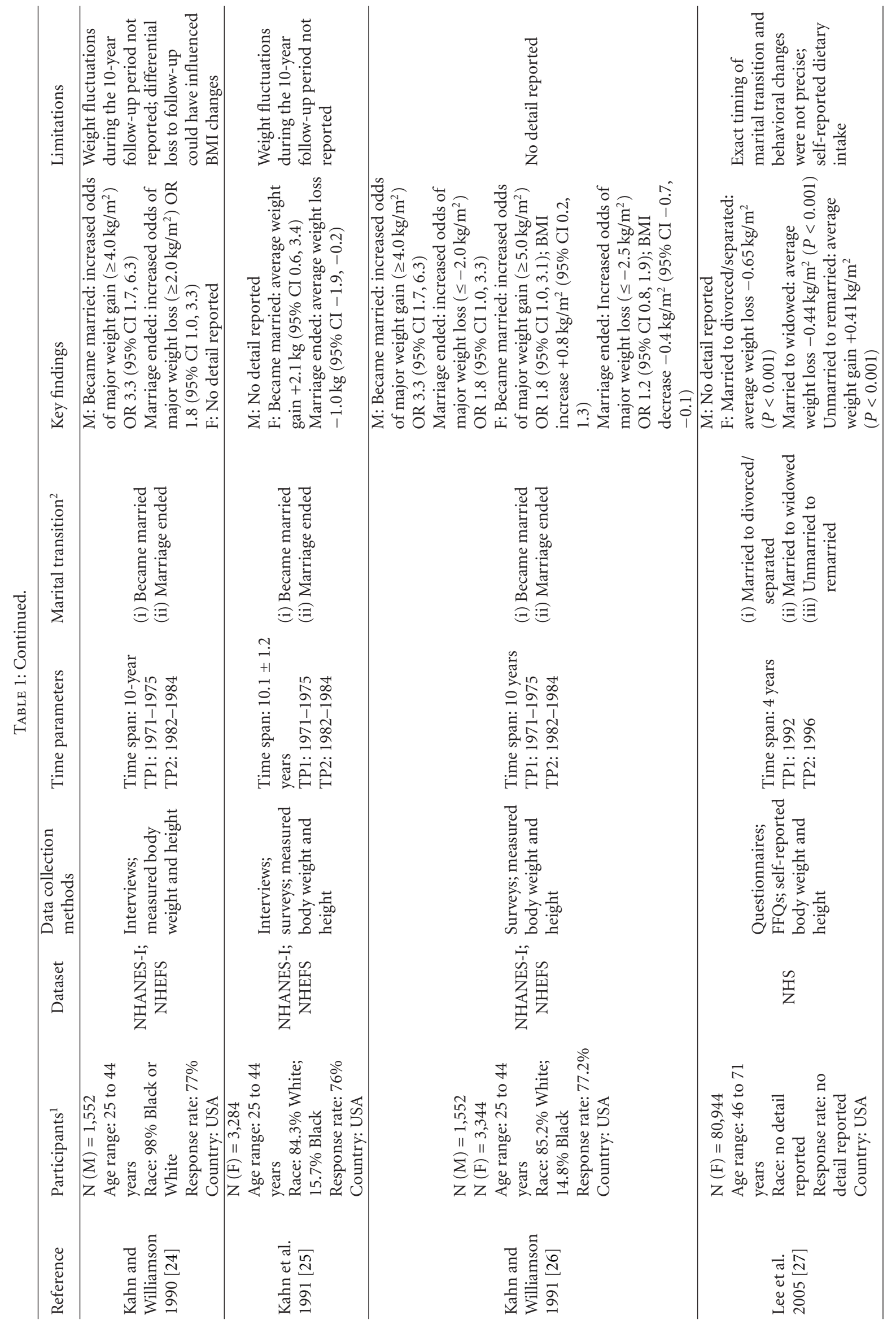




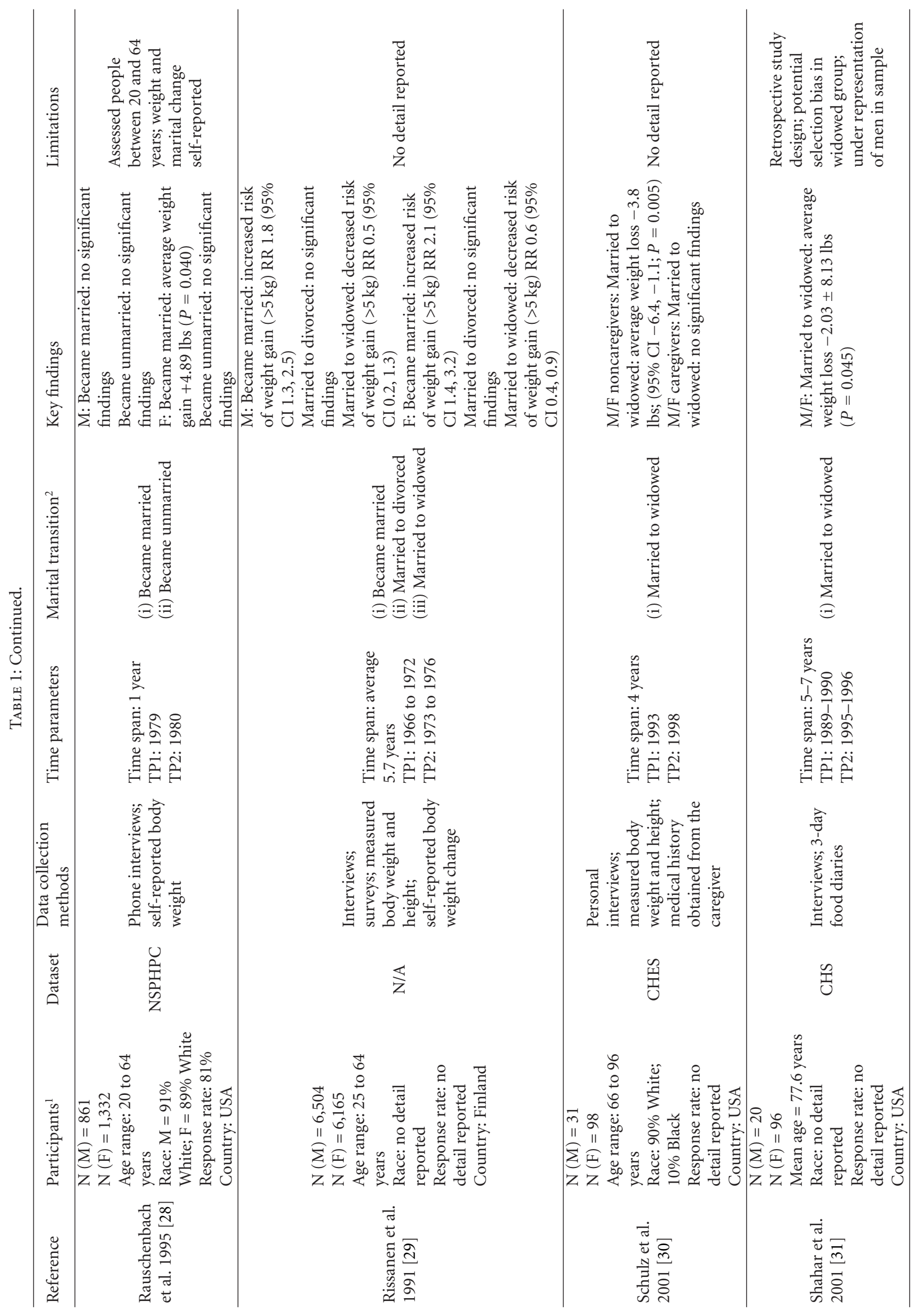




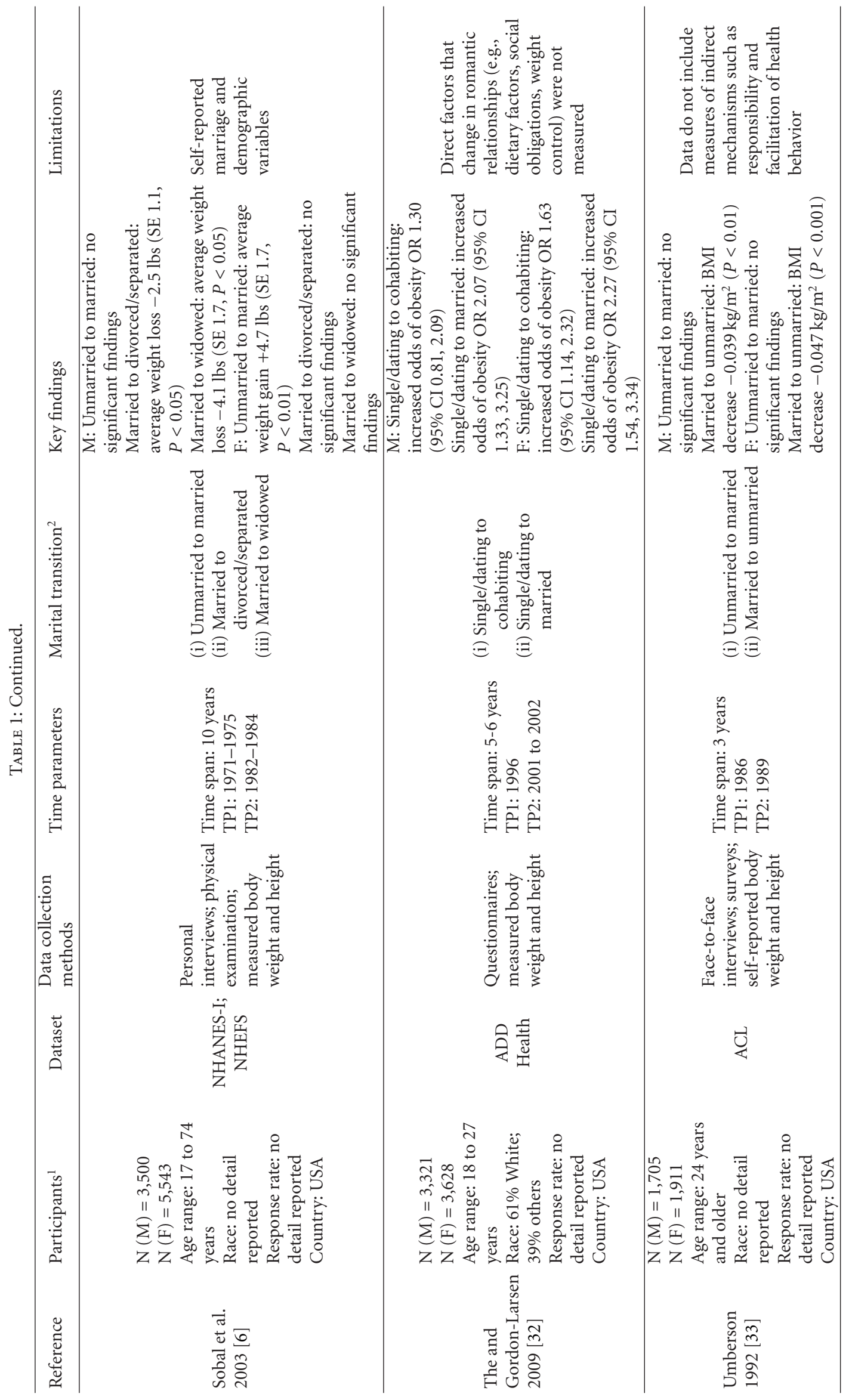




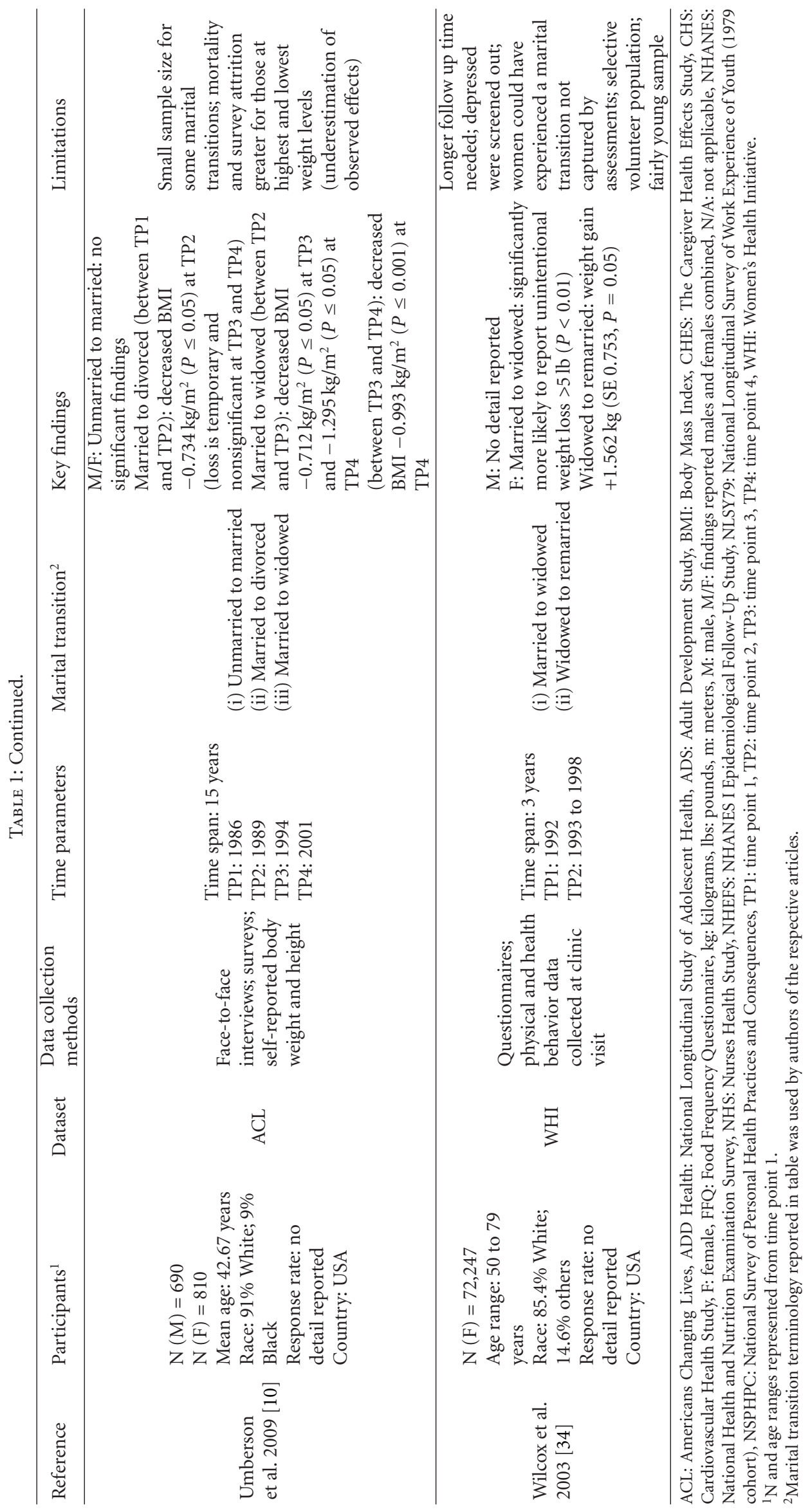




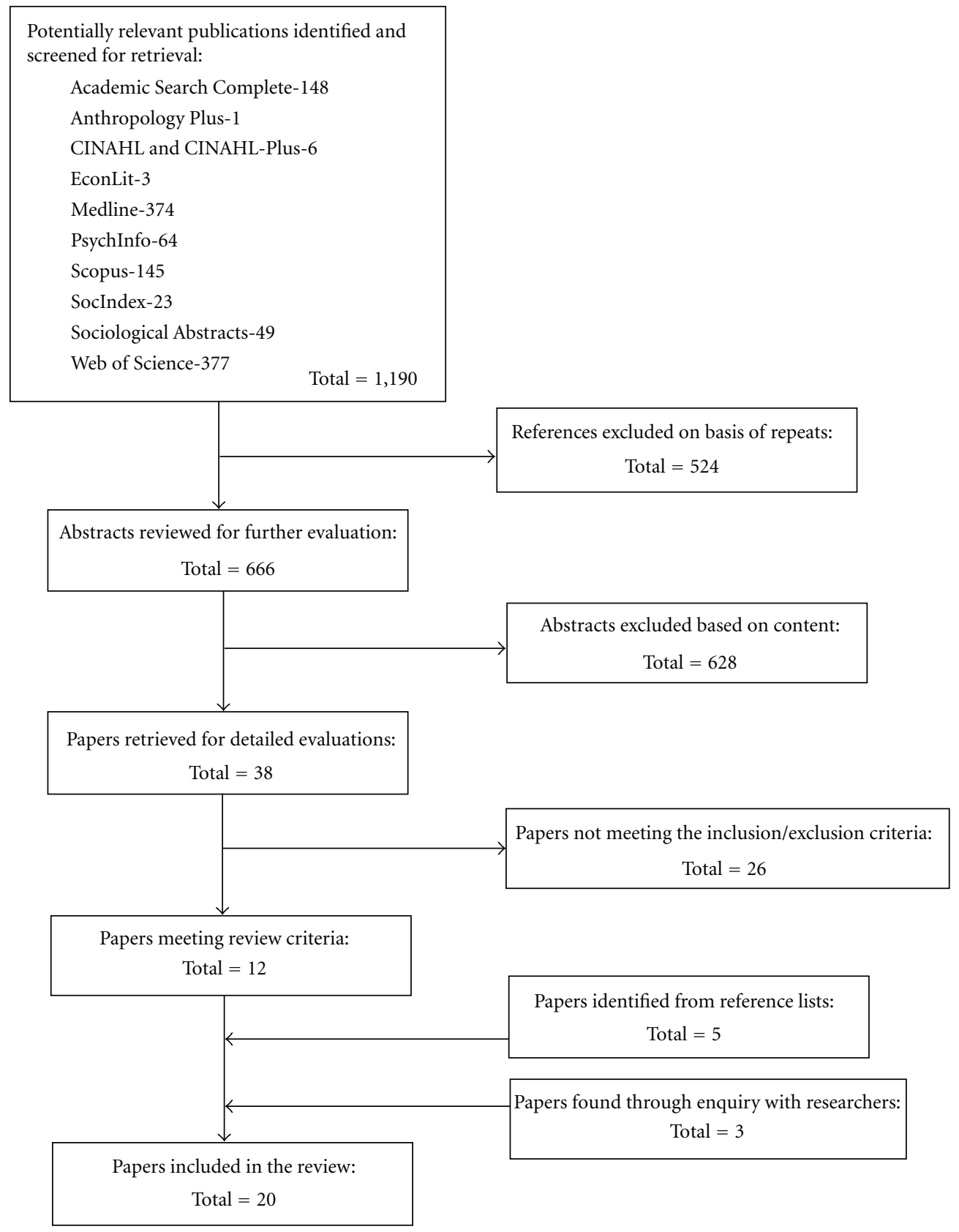

Figure 1: Flow chart of the search process.

though different sample sizes were used for analysis, and each article reports different outcome measures (e.g, weight change, BMI change, major weight gain or loss, etc.). Likewise, Umberson [33] and Umberson et al. [10] both analyzed data from the Americans' Changing Lives (ACL) survey. However, Umberson [33] only included data from Waves I and II, whereas Umberson et al. [10] extended analysis to also include data from Waves III and IV. Both Harris et al. [22] and The and Gordon-Larson [32] report findings from the National Longitudinal Study of Adolescent Health (ADD Health) yet use different waves as baseline data, as well as different comparison groups and outcome measures. In total, the 20 articles report on 15 unique surveys or studies.

Four articles described only transitions into marriage and/or cohabitation [17, 19, 22, 32], 2 articles described only transitions out of marriage and/or cohabitation [30, 31], and 14 articles described both $[6,10,18,20,21,23-29$, $33,34]$. Three articles differentiated between marriage and cohabitation $[18,22,32]$, four articles focused explicitly on remarriage or cohabitation following divorce or widowhood $[18,20,27,34]$, and nine articles specifically analyzed divorce 
and/or widowhood $[6,10,18,20,27,29-31,34]$. Of the 20 articles, 16 analyzed U.S. data $[6,10,18,20,22-28,30-$ $34], 3$ were conducted in Europe (Scotland and Finland) $[17,21,29]$, and 1 reported on Australian data [19]. Articles analyzed time spans between baseline and follow-up ranging from 6 months to 23 years. Table 1 provides a summary of the study design, participant characteristics, key findings, and limitations of the articles as reported by the investigators.

For the purposes of organizing the findings, articles analyzing spans of three years or less were grouped as short time spans, four to eight years as medium time spans, and nine or more years as long time spans. Results are reported by marital transition; however, direct comparisons could not be made due to differing time span durations, independent and dependent variables, comparison groups, and the years over which data were collected.

3.1. Transitions into Marriage. Twelve articles examined the association between all transitions into marriage and changes in BMI or weight without distinguishing between marriage, cohabitation, and/or remarriage $[6,10,17,19,21,23-26$, 28, 29, 33]. Unless otherwise indicated, articles defined the transition into marriage as a change from an unmarried to a married state, whereby unmarried could include never married, cohabiting, divorced/separated, and/or widowed. Thus, cohabitation and remarriage were not determined. The length of follow-up represented by these articles span from 6 months to 15 years and present mixed findings by both time and gender.

Among the articles reporting on short time spans, Anderson et al. [17] surveyed 22 Scottish couples approximately three months before and three months after moving in with a partner or spouse (no differentiation was made between cohabitation and marriage). The period between interviews ranged from 6 to 12 months, and during this time, women gained an average of $1.54 \mathrm{~kg}(\mathrm{SD} 1.7, P<0.001)$ and men gained an average of $1.63 \mathrm{~kg}$ (SD 2.7, $P=0.02$ ). However, since a comparison group of never married individuals living alone was not surveyed, it cannot be assumed that the observed weight gain was solely the result of living with a partner or spouse.

Analyzing a sample of 2,436 U.S. respondents from the one-year National Survey of Personal Health Practices and Consequences (NSPHPC), Rauschenbach et al. [28] found that becoming married was significantly associated with weight in women but not in men. For women, those who became married had an average weight gain of $4.89 \mathrm{lb}$ (SE 2.20, $P=0.04)$ more than their counterparts who remained married. In a two-year analysis of 2,528 U.S. women and men from the Healthy Worker Project, Jeffery and Rick [23] observed that becoming married was associated with an average BMI increase of $0.96 \pm 0.30 \mathrm{~kg} / \mathrm{m}^{2}(P<0.01)$ in women and $0.70 \pm 0.24 \mathrm{~kg} / \mathrm{m}^{2}(P<0.01)$ in men, compared to their counterparts with no change in marital status.

Craig and Truswell [19] interviewed 60 Australian couples before marriage and after more than two years of marriage. Couples were not living together upon recruitment, though remarriage was not determined. Over approximately three years, both women and men showed an average $2 \mathrm{~kg}$ weight gain. In addition, there was a 5\% increase in the number of women classified as overweight or obese between baseline and follow-up, and an $8 \%$ increase in the number of men in this category. Again, because a comparison group was not utilized, it cannot be determined if marriage led to the weight gain.

Umberson [33] analyzed 3,616 respondents over three years from the ACL Survey and found that becoming married was not significantly associated with BMI in women or men, compared to their counterparts who remained unmarried. However, it should be noted that Umberson compared those who became married to those who remained unmarried, whereas most other articles used consistently married individuals or those with no marital status change as the comparison group.

In their analysis of 12,669 adult Finns over a medium time span, Rissanen et al. [29] found that both women (RR 2.1; 95\% CI 1.4, 3.2) and men (RR 1.8; 95\% CI 1.3, 2.5) who became married during the four-to-seven-year study period nearly doubled the risk of substantial weight gain (defined as $>5 \mathrm{~kg}$ ) relative to their consistently married counterparts.

Of the six articles reporting on long time spans, four analyzed data from the same study, three of which were authored by similar research teams. Among 4,836 women and men enrolled in the 10-year NHANES I and NHEFS, Kahn et al. [25] observed that women who became married gained a mean of $2.1 \mathrm{~kg}(95 \%$ CI $0.6,3.4)$ more than women who were consistently married. Likewise, when looking at only those participants who had a major weight gain (defined as a BMI increase of $\geq 5.0 \mathrm{~kg} / \mathrm{m}^{2}$ for women and $\geq 4.0 \mathrm{~kg} / \mathrm{m}^{2}$ for men), Kahn and Williamson [24, 26] found that the risk of major weight gain was significantly associated with becoming married for both women (OR 1.8; 95\% CI 1.0, 3.1 ) and men (OR 3.3; 95\% CI 1.7, 6.3), compared to their consistently married counterparts.

Similarly, Kahn and Williamson [26] reported a significant mean BMI increase of $0.8 \mathrm{~kg} / \mathrm{m}^{2}$ (95\% CI $0.2,1.3$ ) more in women who became married during the 10 -year time period compared to women who were consistently married over the same time period. The direction of the relationship was the same for men; however the results were not significant. Using a much larger sample of 9,403 from the same study, Sobal et al. [6] also found that transitions into marriage (but not remarriage) were significantly associated with weight gain among women, but not men. Women who transitioned into marriage had a weight gain of $4.7 \mathrm{lbs}$ (SE 1.7, $P<0.01)$ more than their counterparts who remained married during the 10-year time period.

Fogelholm et al. [21] analyzed a 10-year weight change among 1,143 Finnish men. Living conditions were classified as living alone throughout the study, stopped cohabiting after entry, started cohabiting after entry, and cohabiting throughout the study. Marital status was not determined so it is unclear what percentage of cohabiting men were married. In this sample of men, entering a cohabiting relationship was not significantly related to weight change compared to those who continuously cohabited. Likewise, Umberson et al. [10] reanalyzed data from 1,500 respondents of the ACL Survey, this time extending analysis to a 15 -year 
time period. Results were similar to the original analysis: becoming married (remarriage controlled for in the analysis) was not significantly associated with BMI in women or men, compared to their counterparts who remained unmarried.

\subsection{Specific Transitions into a Marital or Cohabiting Relationship}

3.2.1. Marriage versus Cohabitation. Three articles, representing time spans of 5 to 23 years, examined the separate associations between marriage or cohabitation and changes in BMI or incident obesity, though did not differentiate between cohabitation following a never-married status and cohabitation following divorce or widowhood [18, 22, 32]. The two articles reporting on medium time spans found no significant relationship between beginning to cohabit and either BMI [22] or incident obesity [32] in women or men, however, did find a significant relationship between becoming married, increased BMI [22], and higher odds of incident obesity [32]. The article reporting on a long time span found separate and significant relationships between both beginning to cohabit and becoming married, and increased BMI in men and women [18].

\subsubsection{Remarriage or Cohabitation Following Divorce or Wid-} owhood. Four articles, analyzing time spans of 3 to 23 years, described the specific associations between remarriage or cohabitation following divorce or widowhood and BMI or weight $[18,20,27,34]$. Both medium and long time span articles found that men's BMI increases after remarrying [20] or cohabiting following divorce [18]. However, inconsistent results were found in women. Among the short and medium time span articles, one reported that women who remarried experienced a BMI increase after four years [27], yet threeyear findings were not significant among widowed women who remarried [34]. Likewise, divorced women who began cohabiting did not exhibit significant changes in BMI in the long time span article by Averett et al. [18].

3.3. Transitions Out of Marriage. Seven articles examined the association between all transitions out of marriage and changes in BMI or weight without distinguishing between divorce or widowhood [17-20, 27, 28, 32]. Unless otherwise indicated, articles defined the transition out of marriage as a change from a married to an unmarried state, whereby unmarried could include cohabiting, divorced/separated, and/or widowed. The length of follow-up represented by these articles span from 1 to 10 years.

Among the articles reporting on short time spans, Rauschenbach et al. [28] reported no significant association between becoming unmarried and weight change in women or men over a one-year time span. However, Jeffery and Rick [23] found that women, but not men, who ended a marriage over the two-year period showed a significant BMI decrease of $0.63 \pm 0.27 \mathrm{~kg} / \mathrm{m}^{2}(P<0.01)$ compared to their counterparts who had no change in marital status. Likewise, Umberson [33] found that the shift from married to unmarried during a three-year span was associated with a significant BMI decrease of $0.047 \mathrm{~kg} / \mathrm{m}^{2}(P<0.001)$ in women and $0.039 \mathrm{~kg} / \mathrm{m}^{2}(P<0.01)$ in men compared to their counterparts who remained married.

Similarly, in the long time span articles by Kahn and Williamson [24, 26] and Kahn et al. [25], transitions out of marriage were associated with weight loss for both women and men. For women whose marriage ended over the 10-year period, mean weight decreased by $1 \mathrm{~kg}(95 \%$ CI $-1.9,-0.2)$ and mean BMI decreased by $0.4 \mathrm{~kg} / \mathrm{m}^{2}(95 \%$ CI $-0.7,-0.1)$ relative to women who remained consistently married [25, 26]. For men, a 10-year change in mean BMI following the ending of a marriage was not significant, however, the risk of major weight loss (defined as a BMI decrease of $\geq 2.0 \mathrm{~kg} / \mathrm{m}^{2}$ ) was significantly associated with ending a marriage (OR 1.8; $95 \%$ CI 1.0, 3.3) [24, 26]. Results reported by Fogelholm et al. [21] support these findings. For men aged 50 to 59 years who transitioned out of a cohabiting relationship during the 10year period, weight decreased by $2.89 \mathrm{~kg}$ (SE $1.36, P=0.03$ ) compared to their counterparts who continuously cohabited. This association was not significant for younger or older men in this sample.

\subsection{Specific Transitions out of Marriage}

3.4.1. Divorce or Separation. Six articles, analyzing time spans of 4 to 23 years, described the relationship of divorce or separation on changes in BMI or weight $[6,10,18,20$, 27, 29]. Articles reporting on medium time spans found a significant association between divorce/separation and BMI loss in women and men $[20,27]$, and no significant association between divorce and weight gain [29]. Among the long time span articles, Sobal et al. [6] reported no significant association between divorce/separation and weight. These trends are supported by Umberson et al. [10] and Averett et al. [18] who found a significant, yet temporary association between divorce and BMI loss in women and/or men, with weight reversals observed after three years post-divorce or separation.

3.4.2. Widowhood. Eight articles, representing time spans of 3 to 15 years, described the relationship of spousal death on BMI or weight changes $[6,10,20,27,29-31,34]$. With the exception of women in the article by Sobal et al. [6], findings from all small, medium, and long time span articles support a significant association between transitions into widowhood and weight or BMI loss in women and men $[6,10,20,27,29-31,34]$. Of note, this association was more pronounced among recently widowed women $(\leq 1$ year) than among longer term widowed women ( $>1$ year) [34], and among widows who had not provided caregiving assistance to their spouse than among widows who had helped their spouse with one or more activities of daily living [30].

\section{Discussion}

Overall, transitions into marriage appear to be associated with weight gain $[6,17-20,22-29,32]$, whereas transitions out of marriage are associated with weight loss $[6,10$, 
18, 20, 21, 23-27, 29-31, 33, 34]. Further analysis by specific marital transition, as well as stratification by gender, reveals similar patterns. Several authors suggest that weight gain after marriage or cohabitation may occur because of increased opportunities for eating due to shared, regular meals and larger portion sizes, as well as decreased physical activity and a decline in weight maintenance for the purpose of attracting an intimate partner [6, 18, 23, 32]. Married individuals are also less likely to smoke and more likely to quit smoking, and smoking cessation is associated with weight gain $[6,18]$. Transitions out of marriage may lead to weight and dietary changes as a result of changes in social support, social control, stress, and depression $[6,20$, $27,33]$. For example, widowers in the study by Wilcox et al. [34] reported substantially higher rates of depression and poorer social functioning, and Shahar et al. [31] found that widowed individuals had less food enjoyment. These psychosocial factors deserve a closer examination in future studies.

4.1. Time Span Duration. The time span between baseline and follow-up greatly varied between the articles, and although significant findings were seen in short, medium, and long time span articles, it is difficult to draw definitive conclusions on the association between marital transitions and weight. The duration of study periods ranged from 6 months to 23 years, and while several articles reported multiple follow-up measurements, most compared changes in weight at two points in time. Results from two articles suggest that weight changes are temporary following a marital transition $[10,18]$. Articles covering short time spans, or those with only one follow-up measurement, were not designed to determine such fluctuations [6, 17, 20-34]. Most articles analyzing long time spans did not identify participants who experienced multiple transitions, which may alter weight and dietary trajectories [6, 21, 24-26].

4.2. Measures. Another challenge to interpreting the results lies in how the main independent and dependent variables were conceptualized, defined, and measured. To start, marital status choices differed between articles. For example, Rauschenbach et al. [28] asked participants to choose between never married, married, widowed, divorced, or separated. Within the never married and divorced groups, Averett et al. [18] further identified participants who were cohabiting with a partner. More specific still is the article by The and Gordon-Larsen [32] that asked unmarried participants to report whether they were single (not in a romantic relationship), dating (not cohabiting), and cohabiting.

In addition, the marital transitions studied were not always defined consistently across articles. Twelve articles grouped all transitions into marriage together $[6,10,17$, 19, 21, 23-26, 28, 29, 33], while six distinguished between marriage, cohabitation, and/or remarriage [18, 20, 22, 27, 32, $34]$. Likewise, seven articles grouped together all transitions out of marriage [21, 23-26, 28, 33], whereas nine examined divorce and widowhood separately $[6,10,18,20,27,29-$ $31,34]$. There was variety even within specified transitions, as seen in the example of remarriage. While Eng et al. [20] and Lee et al. [27] analyzed the transition into remarriage (both divorced/separated to married and widowed to married), Wilcox et al. [34] focused only on remarriage in widowers and Averett et al. [18] analyzed divorced, but cohabiting participants. Again, we acknowledge that grouping never married, divorced/separated, and widowed individuals together in an unmarried category complicates the interpretation of results, as transitions from or into each of these marital states may bring about different behavioral, emotional, and social changes that can affect weight.

Covariates that could affect weight status and weight change also differed among articles, and this variability makes synthesizing and interpreting results challenging. While all articles considered age and gender, only half controlled for income $[6,10,18,22,24-26,28,33,34]$, eight did not control for race or ethnicity $[17,19-21,23$, $27,29,31]$, and five did not control for education level [2022, 27, 31]. Likewise, while 16 articles included a measure of physical activity $[6,19-27,29-34]$, only 12 considered smoking status $[6,20-22,24-27,29,31,33,34]$ and 8 measured dietary intake [19-21, 23, 27, 29, 31, 34]. Other potential confounders, such as health status [20-22, 27, 29$31,34]$, depression $[22,30,31,34]$, and neighborhood-level factors $[6,18,22,24-26,28]$ were each controlled for by less than half of the articles.

Of particular note, articles differed in how they controlled for pregnancy and parity. Of the 14 articles that included premenopausal women, 8 excluded from analysis women who were pregnant at baseline, follow-up, and/or during the study period $[6,17,22,23,25,26,29,32], 1$ controlled for pregnancy [18], and 5 were either unable or did not report excluding or controlling for pregnant women $[10,19,27,28,33]$. In addition, only eight articles considered a woman's parity [6, 18, 22, 25, 26, 28, 29, 33], and parental status was measured in a variety of ways. For example, Harris et al. [22] and Rissanen et al. [29] controlled for births reported during the study period, while Sobal et al. [6], Kahn et al. [25], and Kahn and Williamson [26] controlled for the total number of births and Rauschenbach et al. [28] controlled for the presence of children at home.

Outcome measures also varied across articles. Half of the articles analyzed measured participants' heights and weights $[6,17,24-26,29-32,34]$, while the other half relied on selfreported data $[10,18-23,27,28,33]$, which tend to provide an underreporting of weight and overreporting of height [35]. In addition, the dependent variable differed between weight change $[6,17,19,21,25,28-31,34]$, BMI change $[10,18,20,22-24,26,27,33]$, weight gain or weight loss $[6,28]$, weight gain or weight loss of a specific number of kilograms or pounds $[25,29,34]$, and incident overweight or obesity $[19,32]$, among others. It is difficult to accurately compare these different weight-based outcomes. However, we can note patterns in direction, and overall it appears that transitions into marriage are associated with weight gain and transitions out of marriage are associated with weight loss for both genders. 
4.3. Comparison Groups. Results between articles are difficult to compare due to the use of different comparison groups. The most disparate example of such inconsistencies can be found in the analysis of transitions into marriage or cohabitation (but not remarriage). Those who became married or began cohabiting were compared to participants who transitioned from single (defined as not in a romantic relationship) to dating (defined as in a romantic relationship but not living together) or remained dating in the article by The and Gordon-Larsen [32], while Umberson et al. [10] compared to those who remained unmarried (either never married, divorced, or widowed), and Averett et al. [18] used those who were never married and not cohabiting as the comparison group. Although there is overlap between these three comparison groups, some are more inclusive than others. In addition, the articles by Anderson et al. [17] and Craig and Truswell [19] did not have a comparison group. It is, therefore, difficult to draw definitive conclusions across these articles, even though they all assessed the same marital transition and showed a similar pattern towards weight gain following marriage or cohabitation (with the exception of Umberson et al. [10] who found no significant relationship).

4.4. Years of Data Collection. Finally, it must be mentioned that while our inclusion criteria included articles published from 1990 to present, the data for these articles were obtained over a 40-year time span, from as early as 1966 to as recent as 2004. The percentage of married Americans during this time decreased from $67.6 \%$ in 1960 to $53.6 \%$ in 2010 [36], while the number of cohabitating couples has increased more than 15-fold during this time [13]. In 2009, about $10 \%$ of opposite-sex couples that lived together were not married [37]. Adults are waiting between five and six years longer to get married than 50 years ago [38]. Similar patterns have been observed in Australia [39], Finland [40, 41], and Scotland [42].

Likewise, dietary intake and physical activity patterns have also changed during this time. A review by French et al. [43] reports that, since the 1970s, Americans have dramatically increased intake of soft drinks and foods prepared outside the home, which are often high in added sugar and fat, respectively. Portion sizes have increased over time, whereas food cost as a percentage of income has declined, contributing to over-consumption [43]. Furthermore, sedentary activities such as television and computer use have increased since the 1960s, and an increase in automobile use means that Americans are less likely than in previous years to walk or bike for transportation [43].

Taken together, it is possible that the relationship between marital transition and weight change has shifted over time as both period and cohort effects of marriage, diet, and physical activity may be operating in the data. Although significant results were found in articles analyzing both earlier and more recent data, we cannot determine whether the magnitude of these changes varies across the years, or to what extent these changes are associated with shifting period and cohort effects, as these effects cannot be clearly separated.
4.5. Limitations. There are several limitations to this review. First, because of variations in analytic design and duration, measures, and comparison groups, a quantitative analysis of the evidence was not possible. Next, since most articles report U.S. data, the findings might not be generalizable to other countries where marital patterns may differ. In addition, results may not be generalizable to all couples within the U.S., as studies either recruited only heterosexual couples or did not ask participants about the sex of their partners. Due to the limited number of articles that stratified results beyond gender, it is difficult to determine whether patterns differ by race, ethnicity, age, income, or other sociodemographic characteristics. In addition, many articles did not distinguish cohabitation as a unique marital status, so it is unclear whether marriage, per se, affects weight.

This review is also limited by the relatively small sample size of articles available. Although our initial literature search resulted in over 600 articles, most did not analyze the relationship between a change in marital status and concomitant change in weight or BMI. This is a young but growing topic of research that cannot be clearly summarized at this point in time. However, we believe this systematic review is strengthened by the inclusion of several articles that analyzed nationally representative samples $[6,18,22,24-$ $26,32]$.

\section{Implications}

5.1. For Future Research. As the percentage of U.S. households identifying as unmarried partner households has increased over time [13], so too has the percentage of same-sex couples [44]. However, as this review reveals, the literature currently lacks research about the associations between marital transitions and weight in same-sex couples. Likewise, it is possible that weight changes following marital transitions differ depending on sociodemographic characteristics. New research suggests that weight gain following marriage is most pronounced in newly married women compared to men, and for those who marry at a younger age than those who marry later in life [45]. Further studies are, therefore, needed to explore whether all couples experience similar weight changes after a marital transition, or if these associations differ by an individual's or partner's gender, age, race, ethnicity, income, or geographic location.

In order to better compare the research going forward, it is important that studies utilize similar marital status measures, covariates, and weight outcomes. Additional longitudinal studies with multiple follow-ups can allow researchers to determine if and when weight changes peak, stabilize, and reverse after a marital transition. Furthermore, multiple follow-ups can assist in identifying those individuals who experience more than one marital transition during the study period.

5.2. For Programs, Practice, and Policy. The present review highlights the need to develop and support successful programs aimed at promoting the health benefits and preventing the health detriments related to marital transitions. Taking a life course perspective, children and adolescents should 
receive adequate food and nutrition education in school as an effort to introduce and improve food preparation skills prior to adulthood, cohabitation, and marriage. A recent call for the revival and modernization of home economics courses reveals the need to teach students how to choose and prepare food as part of a comprehensive, school-based obesity prevention effort [46]. This comprehensive effort should also include daily quality physical education and adequate opportunities for all students to participate in physical activity outside of physical education courses, such as recess periods for elementary students, interscholastic sports, and walk- or bicycle-to-school programs [47]. Later on in adulthood, transitions into cohabitation or marriage provide opportunities to engage people in adopting healthier behaviors through food and nutrition intervention programs specifically designed for couples and the newly shared household environment. Such programs have shown promise in improving both obesity-related dietary and physical activity behaviors $[48,49]$.

From a practice standpoint, health providers and marriage professionals_-including physicians, nurse practitioners, registered dietitians, psychologists, social workers, marriage counselors, family therapists, and others-should gather information regarding marital transitions and the health histories of partners. For those transitioning into marriage or cohabitation, health providers and marriage professionals could then target their counseling to promote healthy behaviors, as well as refer patients to couples-based programs. Transitions out of marriage can serve as a flag to probe for symptoms of stress, depression, dietary changes, and weight loss. Referrals can also be provided for services seeking to prevent or treat these symptoms.

From a policy perspective, several states provide incentives for marriage preparation, such as waiving or reducing the marriage license fee for couples that complete a premarital education course [50]. In addition to including communication and conflict management skills, these courses highlight an opportunity to incorporate weight management techniques, food preparation methods, and tips for including physical activity into daily life. Similarly, marriage counseling programs aimed at strengthening marital relationships for temporary assistance for needy families (TANFs) recipients and low-income populations are available in some states [50] and could be broadened to promote healthy weights within couples and families. Finally, many states mandate education for divorcing couples-particularly for couples with children-to discuss parenting issues and the effects of divorce on children [50]. Again, these programs could also review how to maintain healthy habits during the transition out of marriage.

Furthermore, policies and programs should be enhanced to provide the necessary social and emotional support for the aging population. As this review has highlighted, transitions into widowhood-often experienced later in life-are associated with weight loss, possibly as a result of changes in social support, stress, and depression. While programs such as Meals on Wheels and senior centers help to decrease isolation, malnutrition, and food insecurity in this population, adequate and consistent funding and support is required for these and other services as Americans live longer and the number of older adults continues to grow.

In conclusion, this review has found that marital transitions are associated with weight in both men and women. Although additional research is warranted to better understand this phenomenon and its impact on obesity and related behaviors, this review highlights potential opportunities to incorporate effective programs, practices, and policies which aim to promote and support healthy weights and lifestyles upon entering or leaving a marriage or cohabiting relationship.

\section{Conflict of Interests}

The authors declare no conflict of interests.

\section{References}

[1] K. M. Flegal, D. Carroll, B. K. Kit, and C. L. Ogden, "Prevalence of obesity and trends in the distribution of body mass index among US adults, 1999-2010," Journal of the American Medical Association, vol. 307, no. 5, pp. 491-497, 2012.

[2] "Vital signs: state-specific obesity prevalence among adultsUnited States, 2009," Morbidity and Mortality Weekly Report, vol. 59, no. 30, pp. 951-955, 2010.

[3] B. Caballero, "The global epidemic of obesity: an overview," Epidemiologic Reviews, vol. 29, no. 1, pp. 1-5, 2007.

[4] World Health Organization fact sheet: Obesity and Overweight, http://www.who.int/mediacentre/factsheets/fs311/en/, 2011.

[5] K. M. Flegal, B. I. Graubard, D. F. Williamson, and M. H. Gail, "Cause-specific excess deaths associated with underweight, overweight, and obesity," Journal of the American Medical Association, vol. 298, no. 17, pp. 2028-2037, 2007.

[6] J. Sobal, B. Rauschenbach, and E. A. Frongillo, "Marital status changes and body weight changes: a US longitudinal analysis," Social Science and Medicine, vol. 56, no. 7, pp. 1543-1555, 2003.

[7] R. B. Schafer and P. M. Keith, "Influences on food decisions across the family life cycle," Journal of the American Dietetic Association, vol. 78, no. 2, pp. 144-148, 1981.

[8] R. B. Schafer, "Factors affecting food behavior and the quality of husbands' and wives' diets," Journal of the American Dietetic Association, vol. 72, no. 2, pp. 138-143, 1978.

[9] J. Sobal and K. L. Hanson, "Marital stauts, marital history, body weight, and obesity," Marriage and Family Pastor, vol. 47, pp. 474-504, 2011.

[10] D. Umberson, H. Liu, and D. Powers, "Marital status, marital transitions, and body weight," Journal of Health and Social Behavior, vol. 50, no. 3, pp. 327-343, 2009.

[11] L. J. Waite, "Does marriage matter?" Demography, vol. 32, no. 4, pp. 483-507, 1995.

[12] Z. Wu and R. Hart, "The effects of marital and nonmarital union transition on health," Journal of Marriage and Family, vol. 64, no. 2, pp. 420-432, 2002.

[13] W. B. Wilcox and E. Marquardt, The State of Our Unions: Marriage in America 2010, http://stateofourunions.org/2010/SOOU2010.pdf, 2011.

[14] Center for Disease Control, "Marriage and cohabitation in the United States: a statistical portrait based on cycle 6 (2002) 
of the National Survey of Family Growth," Vital and Health Statistics, no. 28, pp. 1-45, 2010.

[15] K. Williams and D. Umberson, "Marital status, marital transitions, and health: a gendered life course perspective," Journal of Health and Social Behavior, vol. 45, no. 1, pp. 8198, 2004.

[16] M. E. Dupre, A. N. Beck, and S. O. Meadows, "Marital trajectories and mortality among US adults," American Journal of Epidemiology, vol. 170, no. 5, pp. 546-555, 2009.

[17] A. S. Anderson, D. W. Marshall, and E. J. Lea, "Shared lives-an opportunity for obesity prevention?" Appetite, vol. 43, no. 3, pp. 327-329, 2004.

[18] S. L. Averett, A. Sikora, and L. M. Argys, "For better or worse: relationship status and body mass index," Economics and Human Biology, vol. 6, no. 3, pp. 330-349, 2008.

[19] P. L. Craig and A. S. Truswell, "Dynamics of food habits of newly married couples: weight and exercise patterns," Australian Journal of Nutrition \& Dietetics, vol. 47, no. 2, pp. 42-46, 1990.

[20] P. M. Eng, I. Kawachi, G. Filzmaurice, and E. B. Rimm, "Effects of marital transitions on changes in dietary and other health behaviours in US male health professionals," Journal of Epidemiology and Community Health, vol. 59, no. 1, pp. 56-62, 2005.

[21] M. Fogelholm, U. Kujala, J. Kaprio, and S. Sarna, "Predictors of weight change in middle-aged and old men," Obesity Research, vol. 8, no. 5, pp. 367-373, 2000.

[22] K. M. Harris, H. Lee, and F. Y. DeLeone, "Marriage and health in the transition to adulthood: evidence for African Americans in the add health study," Journal of Family Issues, vol. 31, no. 8, pp. 1106-1143, 2010.

[23] R. W. Jeffery and A. M. Rick, "Cross-sectional and longitudinal associations between body mass index and marriage-related factors," Obesity Research, vol. 10, no. 8, pp. 809-815, 2002.

[24] H. S. Kahn and D. F. Williamson, "The contributions of income, education and changing marital status to weight change among US men," International Journal of Obesity, vol. 14, no. 12, pp. 1057-1068, 1990.

[25] H. S. Kahn, D. F. Williamson, and J. A. Stevens, "Race and weight change in US women: the roles of socioeconomic and marital status," American Journal of Public Health, vol. 81, no. 3, pp. 319-323, 1991.

[26] H. S. Kahn and D. F. Williamson, "Is race associated with weight change in US adults after adjustment for income, education, and marital factors?" American Journal of Clinical Nutrition, vol. 53, no. 6, 1991.

[27] S. Lee, E. Cho, F. Grodstein, I. Kawachi, F. B. Hu, and G. A. Colditz, "Effects of marital transitions on changes in dietary and other health behaviours in US women," International Journal of Epidemiology, vol. 34, no. 1, pp. 69-78, 2005.

[28] B. Rauschenbach, J. Sobal, and E. A. Frongillo Jr, "The influence of change in marital status on weight change over one year," Obesity research, vol. 3, no. 4, pp. 319-327, 1995.

[29] A. M. Rissanen, M. Heliovaara, P. Knekt, A. Reunanen, and A. Aromaa, "Determinants of weight gain and overweight in adult Finns," European Journal of Clinical Nutrition, vol. 45, no. 9, pp. 419-430, 1991.

[30] R. Schulz, S. R. Beach, B. Lind et al., "Involvement in caregiving and adjustment to death of a spouse: findings from the caregiver health effects study," Journal of the American Medical Association, vol. 285, no. 24, pp. 3123-3129, 2001.

[31] D. R. Shahar, R. Schultz, A. Shahar, and R. R. Wing, "The effect of widowhood on weight change, dietary intake, and eating behavior in the elderly population," Journal of Aging and Health, vol. 13, no. 2, pp. 186-199, 2001.

[32] N. S. The and P. Gordon-Larsen, "Entry into romantic partnership is associated with obesity," Obesity, vol. 17, no. 7, pp. 1441-1447, 2009.

[33] D. Umberson, "Gender, marital status and the social control of health behavior," Social Science and Medicine, vol. 34, no. 8, pp. 907-917, 1992.

[34] S. Wilcox, A. Aragaki, C. P. Mouton, K. R. Evenson, S. Wassertheil-Smoller, and B. L. Loevinger, "The effects of widowhood on physical and mental health, health behaviors, and health outcomes: the women's health initiative," Health Psychology, vol. 22, no. 5, pp. 513-522, 2003.

[35] S. C. Gorber, M. Tremblay, D. Moher, and B. Gorber, "A comparison of direct vs. self-report measures for assessing height, weight and body mass index: a systematic review," Obesity Reviews, vol. 8, no. 4, pp. 307-326, 2007.

[36] U.S. Census Bureau, Current Population Survey-March, and Annual Social and Economic Supplements. Table MS-1. Marital Status of the Population, 15 Years Old and Over by Sex and Race: 1950 to the Present, http://www.census.gov/population/socdemo/hh-fam/ms1.xls, 2011.

[37] U.S. Census Bureau, Newsroom: Families \& Households: Census Bureau Reports Families With Children Increasingly Face Unemployment, http://newsroomwww.census.gov/ releases/archives/families_households/cb10-08.html, 2011.

[38] U.S. Census Bureau, Current Population Survey-March, and Annual Social and Economic Supplements. Table MS-2. Estimated Median Age at First Marriage, by Sex: 1890 to the Present, http://www.census.gov/population/socdemo/hhfam/ms2.xls, 2011.

[39] D. de Vaus, Diversity and Change in Australian Families: Statistical Profiles, Australian Institute of Family Studies, Melbourne, Australia, 2004.

[40] K. E. Joutsenniemi, T. P. Martelin, S. V. Koskinen et al., "Official marital status, cohabiting, and self-rated healthtime trends in Finland, 1978-2001," European Journal of Public Health, vol. 16, no. 5, pp. 476-483, 2006.

[41] Official Statistics of Finland (OSF): Changes in marital status 2010, http://www.stat.fi/til/ssaaty/2010/index_en.html, 2012.

[42] A. Morrison, D. Headrick, F. Wasoff, and S. Morton, Family Formation and Dissolution: Trends and Attitudes among the Scottish Population, Centre for Research on Families and Relationships, Edinburgh,UK, 2004.

[43] S. A. French, M. Story, and R. W. Jeffery, "Environmental influences on eating and physical activity," Annual Review of Public Health, vol. 22, pp. 309-335, 2001.

[44] G. J. Gates, Same-Sex Couples and the Gay, Lesbian, Bisexual Population: New Estimates from the American Community Survey, http://escholarship.org/uc/item/8h08t0zf, 2011.

[45] D. Tumin and Z. Qian, "Marital transitions and weight changes," in Proceedings of the 106th Annual American Sociological Association Annual Meeting, Las Vegas, Nev, USA, August 2011.

[46] A. H. Lichtenstein and D. S. Ludwig, "Bring back home economics education," Journal of the American Medical Association, vol. 303, no. 18, pp. 1857-1858, 2010.

[47] Centers for Disease Control and Prevention, "School health guidelines to promote healthy eating and physical activity," Morbidity and Mortality Weekly Report, vol. 60, no. 5, pp. 2833, 2011.

[48] V. Burke, N. Giangiulio, H. F. Gillam, L. J. Beilin, S. Houghton, and R. A. K. Milligan, "Health promotion in couples adapting 
to a shared lifestyle," Health Education Research, vol. 14, no. 2, pp. 269-288, 1999.

[49] V. Burke, N. Giangiulio, H. F. Gillam, L. J. Beilin, and S. Houghton, "Physical activity and nutrition programs for couples: a randomized controlled trial," Journal of Clinical Epidemiology, vol. 56, no. 5, pp. 421-432, 2003.

[50] K. N. Gardiner, M. E. Fishman, P. Nikolov, A. Glosser, and S. Laud, State policies to promote marriage: final report, September 2002, http://aspe.hhs.gov/hsp/marriage02f/, 2012. 


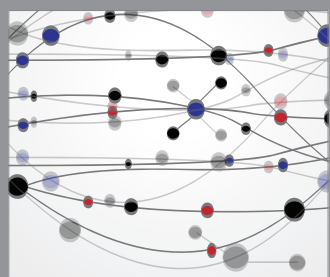

The Scientific World Journal
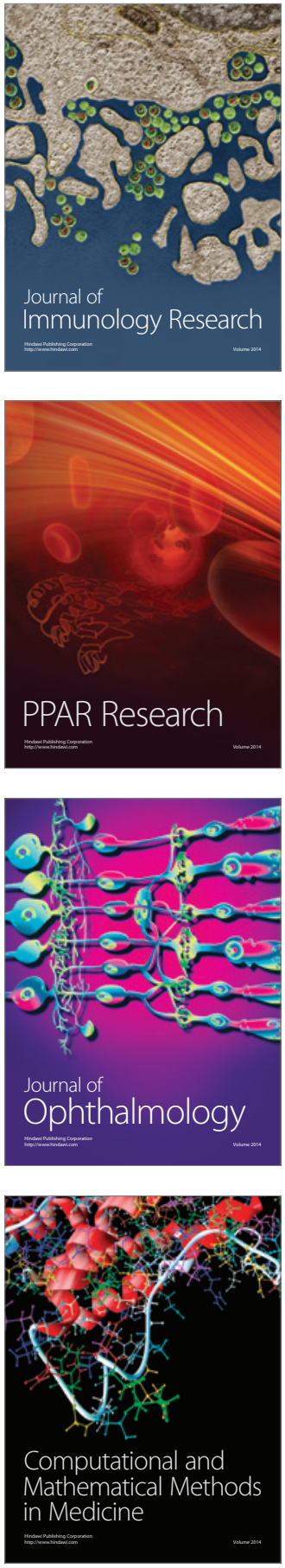

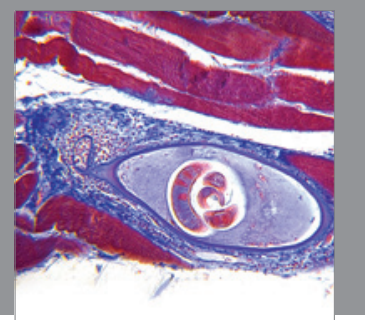

Gastroenterology

Research and Practice
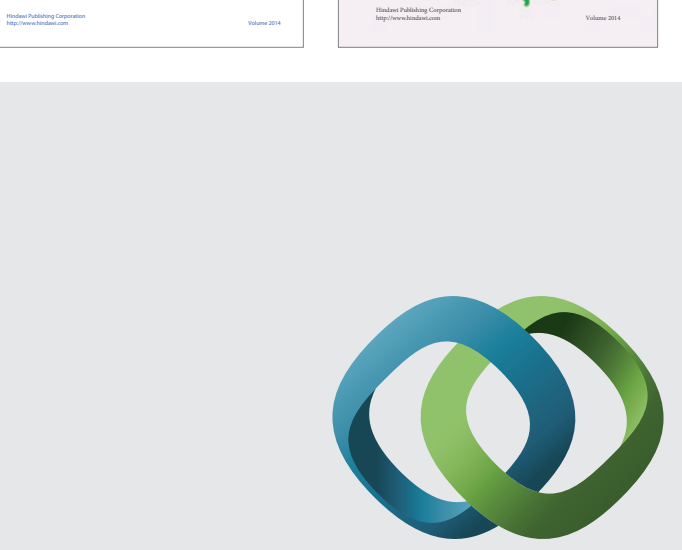

\section{Hindawi}

Submit your manuscripts at

http://www.hindawi.com
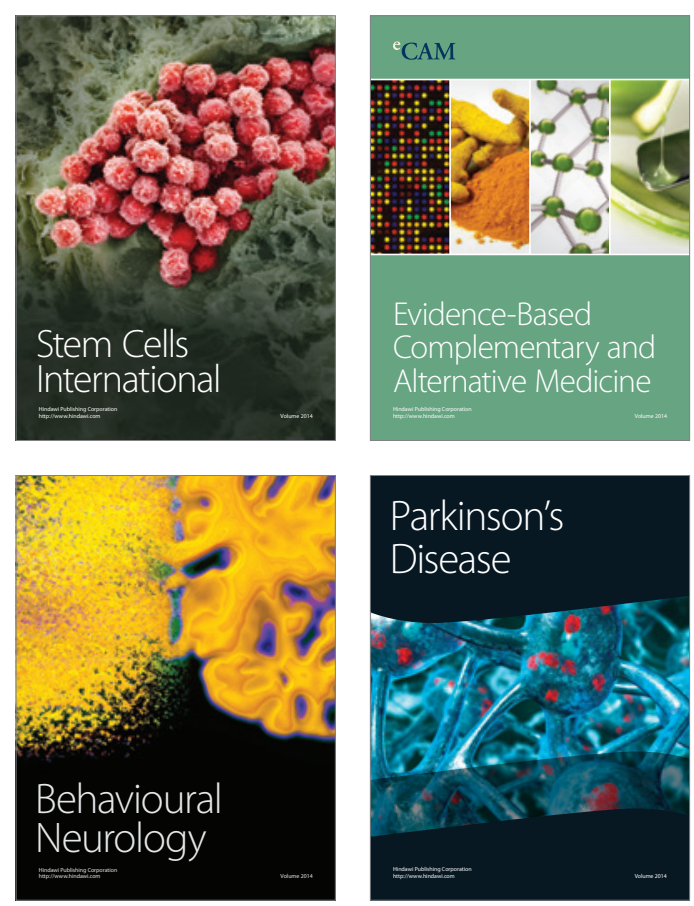

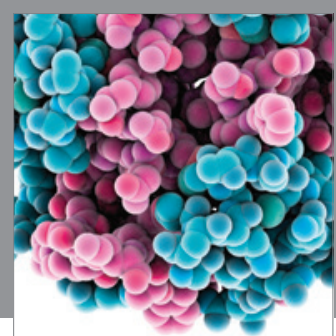

Journal of
Diabetes Research

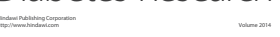

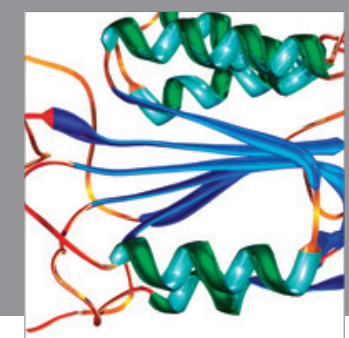

Disease Markers
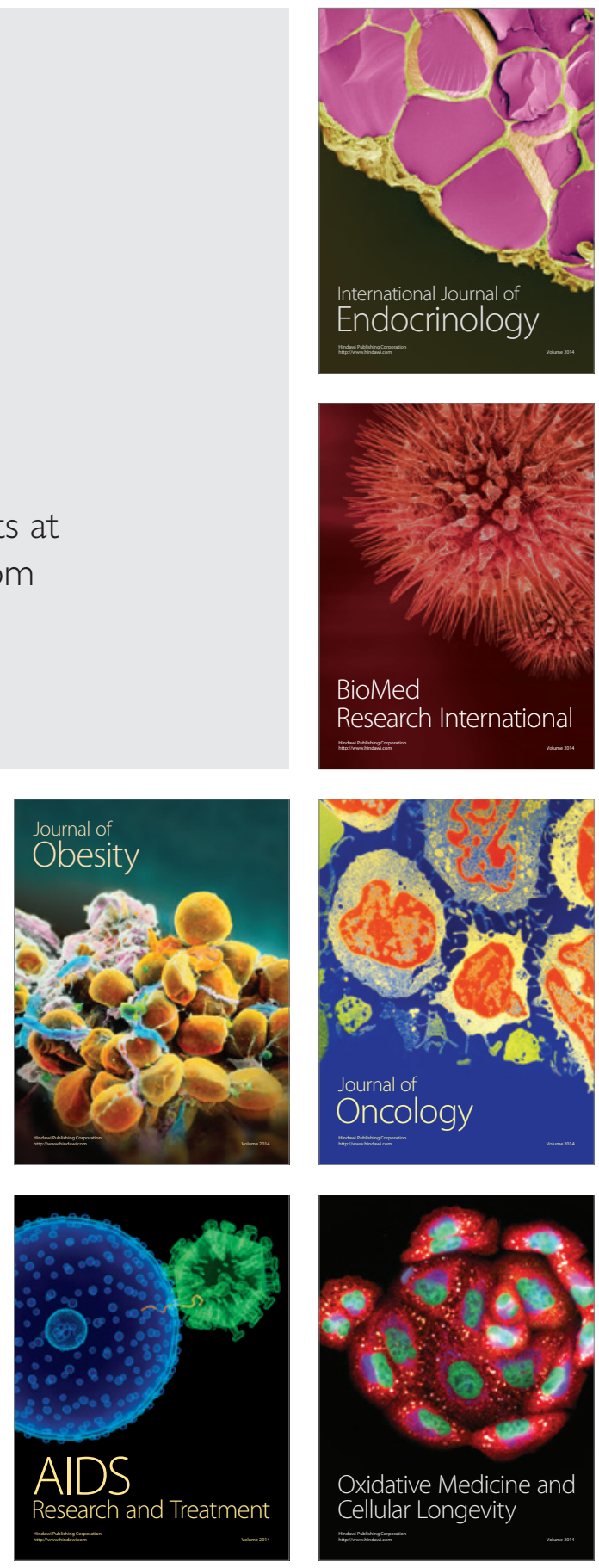